\title{
Weibull- $k$ Revisited: “Tall” Profiles and Height Variation of Wind Statistics
}

\author{
Mark Kelly • Ib Troen · Hans E. Jørgensen
}

Received: 22 April 2013 / Accepted: 10 February 2014 / Published online: 19 March 2014

(C) The Author(s) 2014. This article is published with open access at Springerlink.com

\begin{abstract}
The Weibull distribution is commonly used to describe climatological wind-speed distributions in the atmospheric boundary layer. While vertical profiles of mean wind speed in the atmospheric boundary layer have received significant attention, the variation of the shape of the wind distribution with height is less understood. Previously we derived a probabilistic model based on similarity theory for calculating the effects of stability and planetary boundary-layer depth upon long-term mean wind profiles. However, some applications (e.g. wind energy estimation) require the Weibull shape parameter $(k)$, as well as mean wind speed. Towards the aim of improving predictions of the Weibull- $k$ profile, we develop expressions for the profile of long-term variance of wind speed, including a method extending our probabilistic wind-profile theory; together these two profiles lead to a profile of Weibullshape parameter. Further, an alternate model for the vertical profile of Weibull shape parameter is made, improving upon a basis set forth by Wieringa (Boundary-Layer Meteorol, 1989, Vol. 47, 85-110), and connecting with a newly-corrected corollary of the perturbed geostrophic-drag theory of Troen and Petersen (European Wind Atlas, 1989, Ris $\varnothing$ National Laboratory, Roskilde). Comparing the models for Weibull- $k$ profiles, a new interpretation and explanation is given for the vertical variation of the shape of wind-speed distributions. Results of the modelling are shown for a number of sites, with a discussion of the models' efficacy and applicability. The latter includes a comparative evaluation of Wieringa-type empirical models and perturbed-geostrophic forms with regard to surface-layer behaviour, as well as for heights where climatological wind-speed variability is not dominated by surface effects.
\end{abstract}

Keywords Atmospheric stability · Boundary-layer depth · Weibull statistics · Wind energy $\cdot$ Wind profiles

M. Kelly $(\varangle) \cdot$ I. Troen · H. E. Jørgensen

Wind Energy Division/Meteorology Group, Risø Campus of the Danish Technical University,

Frederiksborgvej 399, 4000 Roskilde, Denmark

e-mail: mkel@dtu.dk 


\section{Introduction}

To better predict multi-year wind distributions at heights well beyond the atmospheric surface layer ('ASL' hereafter), i.e. above 50-100 m, based on measurements at lower heights, the extrapolation of measured statistics demands a model for not only the wind profile but also model(s) for the profile(s) of the long-term wind statistics. Such models have become more desirable so far as application is concerned; one example is wind resource assessment, driven by the ever-increasing heights above the Earth's surface at which turbines are being mounted. Commercially available lidar devices are able to measure wind statistics at these heights, but due to the current cost of lidar technology this is generally not feasible; this is particularly true for observations lasting multiple years, i.e. over time scales needed to reliably characterize the local wind climate.

Models for the scalar wind profile have existed for decades, most notably the similarity theory of Monin and Obukhov (1954). However, such profiles incorporating the effects of atmospheric stability are generally not derived for long-term application. While some power laws have been employed for both 10-min and long-term use, this has been without any physical or solid theoretical relation to stability. Kelly and Gryning (2010) developed a theory for distributions of stability based on surface-layer fluxes, exploiting it to produce a statistical long-term wind-profile model based on Monin-Obukhov ("M-O") theory; this was then generalized (adapted) in order to extend the 'tall' profile model of Gryning et al. (2007) for long-term application. By "long-term," here we mean decades, applicable e.g. to the lifetime of a wind turbine (in contrast with typical 10-min average profiles obtained from measurements, or the time scales of climate models, which can exceed a century or millennium).

Various models for the vertical profiles of parameters describing wind-speed distributions (i.e. Weibull parameters) arose in the 1970s; Justus and Mikhail (1976) made a surface-layer fit to find the behaviour of the wind distribution with height, with Justus et al. (1978) comparing similarly simple models in the surface layer, and Spera and Richards (1979) developing an alternative including a dependence upon surface roughness. Mikhail (1985) showed, however, that these early attempts did not give usable results beyond the the surface layer. Wieringa (1989) found an empirical relation for the vertical profile of the Weibull shape parameter for wind distributions, but it lacks any roughness dependence and contains a dimensional coefficient that varies from site to site in an undetermined manner. In the European Wind Atlas (Troen and Petersen 1989, denoted 'EWA' hereafter), perturbation theory was applied to the geostrophic drag law, along with $\mathrm{M}-\mathrm{O}$ similarity in order to extrapolate observed wind statistics. This gave a coupled formulation for the profiles of Weibull parameters via extrapolation of both first and second moments of wind speed, as influenced by geostrophicscale $^{1}$ stability perturbations in a climatological mean sense. The EWA perturbation theory also included an estimate of the height at which the stability-affected wind variability is minimal, using it to model the height at which the long-term variance of wind speed reaches its minimum; as shown below, this is equivalent to accounting for the height where the wind distribution is narrowest.

In this work we derive several formulations for the vertical profile of the Weibullshape $(k)$ parameter by developing probabilistic forms for the corresponding profile of longterm wind-speed variance, via extension and use of the long-term probabilistic wind-profile model of Kelly and Gryning (2010). We further offer an alternative empirical model for the profile $k(z)$, generalizing the form of Wieringa (1989) and making it self-consistent,

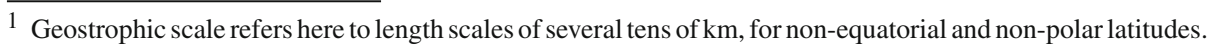


while also adapting it for measurement-based application. This includes correction of the EWA (Troen and Petersen 1989) estimate of the height of narrowest wind distribution (peak of $k$ ), and its incorporation in the adapted Wieringa-like profile for the Weibull shape parameter. We also elucidate some theoretical details and refinement of the EWA methodology to improve upon Troen and Petersen (1989), as well as subsequent practical and theoretical implications; the latter includes a new physical, non-empirical interpretation of the shape of the $k$-profile as related to boundary-layer depth.

\section{Wind Statistics, Theory, and Development}

To describe vertical profiles of wind statistics, we consider the vertical wind-speed distribution. We follow the standard assumption that typically averaged (e.g. 10- to 30-min mean) observations of wind speed, when collected over the long term (years) from a range of sufficiently narrow wind sectors (typically $30^{\circ}$ or less) at a given height $z$ above ground level, can be described by a two-parameter Weibull distribution

$$
p(U)=\frac{k}{U}\left(\frac{U}{A}\right)^{k} \mathrm{e}^{-(U / A)^{k}}
$$

where $p(U)$ is the probability density function of wind speed $U, k$ is the Weibull-shape parameter, and $A$ is the so-called scale parameter. Just as the mean wind speed has a profile $U(z)$, the Weibull parameters can also vary with height; for clarity, in (1) the z-dependence implicit in $U, A$, and $k$ has been omitted. For a wind-speed probability distribution of the Weibull form (Eq. 1), the $n$th moment of the wind speed is given by

$$
\left\langle U^{n}(z)\right\rangle=A^{n} \Gamma(1+n / k)
$$

where $\Gamma(x)$ is the Gamma function, and we use angle-brackets to refer to an average of observations over one or more years. Thus the long-term mean wind speed is equal to $A \Gamma(1+$ $1 / k)$; the long-term mean wind-speed profile $\langle U(z)\rangle$ depends upon the profile $k(z)$ as well as $A(z)$.

From (2), one finds that higher moments of $U(z)$ are more sensitive to $k(z)$ than the mean, with $\partial \ln \left\langle U^{n}\right\rangle / \partial k$ roughly proportional to $n$ (specifically $\partial \ln \left[\left\langle U^{n}\right\rangle\right] / \partial k=-n k^{-2} \chi(1+n / k)$, where the DiGamma function $\chi(x) \sim 1$ for real-world values $0.5<k<10$ ). For example, the expected long-term wind-power density has a normalized $k$-derivative $\partial \ln \left(\left\langle U^{3}\right\rangle\right) / \partial k$ whose magnitude is larger than $|\partial \ln (\langle U\rangle) / \partial k|$ over the range of $k(0<k<5)$ observed in the atmospheric boundary layer (ABL).

Figure 1 illustrates the significance of $k(z)$ in application: for a typical contemporary (megawatt-scale) wind-turbine power curve, we see that a $10 \%$ over-prediction in $k$ can translate into an error in energy prediction exceeding $5 \%$ for a range of mean wind speeds $\gtrsim 8 \mathrm{~m} \mathrm{~s}^{-1}$ and smaller $k$ (which tend to happen farther from the ground), and approaching $-10 \%$ for low-wind sites. This result simply follows from numerically integrating the product of the wind distribution (1) and a power curve.

Thus extrapolation of observed winds to predict the wind distribution (or e.g. wind power) at some height $z$ above the measurement height requires not only a model for the wind profile (or $A$-profile), but also demands a model to obtain $k(z)$ - either directly or via the profile of a higher-order moment as in (2).

In order to obtain e.g. height-dependent estimates of energy production based on observed statistics, for a two-parameter statistical description of the wind field one needs to know the 


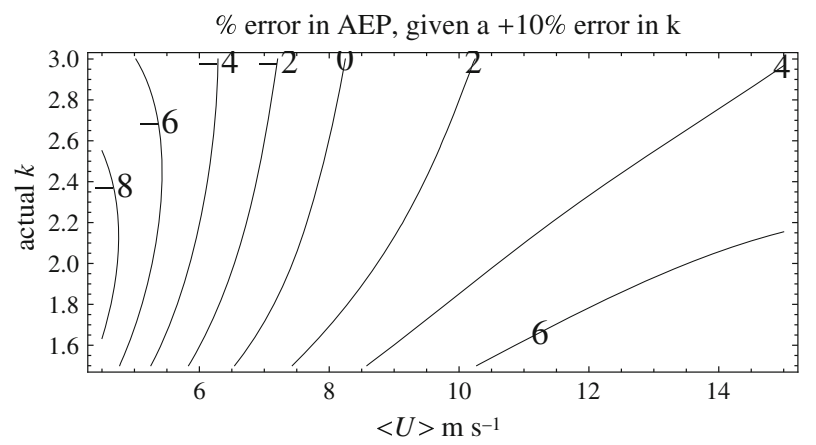

Fig. 1 Contours of the percentage error in estimated wind-power density (as annual energy production, 'AEP') due to a $10 \%$ over-prediction in $k$, as a function of actual $k$ and long-term mean wind speed $\langle U\rangle$; case for typical 1.5 MW turbine power curve shown (General Electric model 1.5xle, see e.g. www.gepower.com or Mathematica example documentation)

profiles of two moments of the wind speed. Along with the mean wind speed (first moment), we consider the second moment of wind speed in our statistical profile modelling. We do this because lower-order statistical moments (relative to higher moments) tend to be less demanding in terms of the amount of data required (less sensitive to the length of the time series used), generally less uncertain (e.g. Lenschow et al. 1994), as well as being more directly relatable to flow dynamics. Further, the use of second and first moments together for the purpose of vertically extrapolating observed Weibull- $k$ has been established in the EWA, (Troen and Petersen 1989) methodology, which has successfully been used in practice within the wind industry for heights up to $80-100 \mathrm{~m}$ over the last couple of decades (Mortensen et al. 2001).

\subsection{Relating Profiles of Long-Term Wind Speed, Variance, and Weibull- $k$ Parameter}

Continuing to use a two-parameter Weibull distribution (1) for the wind speed at a given height $z$, the effects of stability and finite ABL depth (or other influences, if their effects upon the two moments of $U$ are also parametrized) can be translated into a modification of $A(z)$ and $k(z)$. We take the long-term second moment of wind speed $\left\langle U^{2}\right\rangle$ to be comprised of independent first- and second-order contributions,

$$
\left\langle U^{2}\right\rangle=\langle U\rangle^{2}+\sigma_{U}^{2} .
$$

Exploiting (2) by taking the ratio of the second moment to the squared first moment of wind speed and using (3), one obtains

$$
\frac{\left\langle U^{2}\right\rangle}{\langle U\rangle^{2}}=\frac{\Gamma(1+2 / k)}{\Gamma^{2}(1+1 / k)}=1+\frac{\sigma_{U}^{2}}{\langle U\rangle^{2}}
$$

thus eliminating $A$. Around $k=1$ the ratio of Gamma functions in (4) approaches $\left(1+k^{-\sqrt{\pi}}\right)$, which can be made more useful by noting that between $k=0.5$ and 5 (encompassing the range of $k$ generally observed in the $\mathrm{ABL}$ ) the ratio is more closely equal to

$$
\frac{\Gamma(1+2 / k)}{\Gamma^{2}(1+1 / k)} \simeq 1+k^{-1.87}
$$

i.e. within a fraction of a percent. Together, (4) and (5) imply 


$$
k \simeq\left(\frac{\langle U\rangle}{\sigma_{U}}\right)^{1.07}
$$

since $2 / 1.87 \approx 1.07$. Given descriptions of the long-term profiles of wind speed $\langle U\rangle(z)$ and variance $\sigma_{U}^{2}(z),(4)$ and (5) also allow solution for $k$ at some height $z_{\text {pred }}$, given an observed $k\left(z_{\mathrm{obs}}\right)$,

$$
k\left(z_{\text {pred }}\right) \simeq k\left(z_{\text {obs }}\right)\left[\frac{\langle U\rangle\left(z_{\text {pred }}\right) / \sigma_{U}\left(z_{\text {pred }}\right)}{\langle U\rangle\left(z_{\text {obs }}\right) / \sigma_{U}\left(z_{\text {obs }}\right)}\right]^{1.07},
$$

assuming observed and predicted wind distributions are both Weibull (probability mass is conserved).

Wieringa (1989) noted that the shape of $k(z)$ is opposite of the shape of the diurnalcycle component of $\sigma_{U}^{2}(z)$, stating that $k \approx 0.94 A /\left(\sigma_{U}\right)_{\text {diurnal }}$; this is compatible with Eq. 6 since $\langle U\rangle=A \Gamma(1+1 / k)$, or roughly $\langle U\rangle \approx 0.9 A$ for typical $k$. Justus et al. (1978) reported a $k$-relation equivalent to Eq. 5 but with an exponent of 1.086, which we note gives a systematic over-prediction of $k$ for $k<3$, i.e. in most real-world situations. More recently, Akdağ and Dinler (2009) used a third-moment fit and $\langle U\rangle^{3} /\left\langle U^{3}\right\rangle$ to obtain $k$, employing an approximation equivalent to $\Gamma(1+3 / k) / \Gamma^{3}(1+1 / k) \approx[3.69 /(k-1)]^{1 / 2}$, which gives errors of several percent for $k>3$ or $k<1.3$; as stated above, here we attempt only to model the long-term mean and variance of wind speed or the $k$-profile itself.

Since the long-term mean wind speed (without terrain elevation effects) generally increases monotonically up to at least $200 \mathrm{~m}$ with well-behaved (positive) long-term vertical shear $\mathrm{d}\langle U\rangle / \mathrm{d} z$, then for $z \lesssim 200 \mathrm{~m}$ the profile $k(z)$ is typically dictated by the shape of the long-term variance $\sigma_{U}^{2}(z)$. Aside from the effects of terrain, it is generally found that the character of the $\sigma_{U}$ profile (e.g. d $\sigma_{U} / \mathrm{d} z$ ) changes at a height around 100-150 m, which with $U(z)$ leads to a maximum in $k$, giving $k(z)$ its characteristic bowed shape (c.f. Wieringa 1989).

\subsection{Modelling Profiles of Moments of Wind Speed, Towards $k(z)$}

The following two sub-sections present the profiles of long-term mean wind speed and longterm wind variance, which are used to give new models for observation-based $k(z)$.

\subsubsection{Model for Climatological Wind Profile}

As written above, one means of obtaining $k(z)$ involves using the associated mean wind profile $\langle U\rangle(z)$ along with the profile of wind-speed variance. A form for the profile of longterm dimensionless mean wind speed was derived by Kelly and Gryning (2010), based on their model for observed stability distributions. This involved statistical adaptation of $\mathrm{M}-\mathrm{O}$ theory, and generalization of the 'tall' wind profile of Gryning et al. (2007) (which adds the influence of ABL depth $h$ to $\mathrm{M}-\mathrm{O}$ theory through a height-varying friction velocity $u_{*}(z / h)$ and via the geostrophic wind speed $\left.G=\left.U\right|_{z=h}\right)$ to a climatological mean form. For representative (long-term) values $h_{\text {eff }}$ and geostrophic wind speed $G_{\text {eff }}$ (with $z_{0}$ being a mean roughness length, which is implicitly done in common practice), defining $\langle U\rangle\left(h_{\mathrm{eff}}\right)=G_{\text {eff }}$, the dimensionless mean wind profile (Kelly and Gryning 2010) is

$$
\begin{aligned}
& \left\langle\frac{\kappa U}{u_{* 0}}\right\rangle=\ln \left(\frac{z}{z_{0}}\right)-\langle\psi\rangle\left(z, \sigma_{ \pm}, n_{ \pm}\right)-\frac{z}{h_{\mathrm{eff}}}\left[\langle\psi\rangle_{z}\left(z, \sigma_{ \pm}, n_{ \pm}\right)-\langle\psi\rangle\left(z, \sigma_{ \pm}, n_{ \pm}\right)\right] \\
& +\frac{h_{\mathrm{eff}}}{2 \ell_{\mathrm{mid}}^{\mathrm{eff}}}\left[1-\left(1-\frac{z}{h_{\mathrm{eff}}}\right)^{2}\right]+\frac{z}{h_{\mathrm{eff}}}\left(s_{\mathrm{eff}}-1\right)
\end{aligned}
$$


where $u_{* 0}$ is the surface-layer friction velocity, $\kappa=0.4$ is the von Karman constant, $\overline{\langle\psi\rangle_{z}}$ is the long-term stability correction averaged up to height $z$, and $s_{\text {eff }} \equiv \kappa h_{\text {eff }}\left\langle u_{* 0}^{-1} d G / d z\right\rangle$ is the mean dimensionless shear across the ABL. ${ }^{2}$ The stability correction is dependent on the inverse Obukhov length variability ${ }^{3}\left(\sigma_{ \pm}\right)$and fraction of occurrence $\left(n_{ \pm}\right)$for stable $\left(_{+}\right)$ and unstable (_) conditions, respectively, and the coefficient $h_{\text {eff }} / 2 \ell_{\text {mid }}^{\text {eff }}$ is equal to the difference between the dimensionless geostrophic wind $\kappa G_{\text {eff }} / u_{* 0}$ and the profile form (8) evaluated at $z=h_{\text {eff }}$. The long-term dimensionless geostrophic ABL shear is taken to be 1 $\left(s_{\text {eff }} \approx \kappa G_{\text {eff }} /\left\langle u_{* 0}\right\rangle\right)$, equivalent to the neglect of the corresponding baroclinic term by Gryning et al. (2007). With $s_{\text {eff }}=1$, the form (8) basically behaves as a Monin-Obukhov profile, but "bends back" approaching the top of the ABL; i.e. the quadratic term forces the

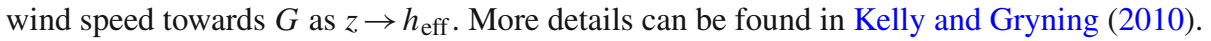

Because we later compare with Weibull- $k$ predictions made by the EWA method (Troen and Petersen 1989), we also include here the effective EWA form for extrapolating measured mean wind speed, since the EWA does not provide an explicit wind profile. Rather, the EWA does a stability-induced perturbation of the logarithmic law and takes the ratio of

$$
\ln \left(\frac{z}{z_{0}}\right)\left\{1+\left[\frac{\Delta u_{*}}{u_{* 0}}\right]_{\text {off }}\right\}+\left(\frac{z}{z_{m}}\right)\left\{\left[\frac{\Delta u_{*}}{u_{* 0}}\right]_{\mathrm{rms}} \ln \left(\frac{z_{m}}{z_{0}}\right)-\psi_{s}\left(\frac{z_{m}}{L_{\mathrm{off}}}\right)-\psi_{u}\left(\frac{z_{m}}{L_{\mathrm{rms}}}\right)\right\}
$$

evaluated at measured and predicted heights, respectively, to be equal to the ratio of measured and predicted mean wind speed $\langle U\rangle$. This ratio is used, along with a ratio of $\sigma_{U}$ at predicted/observed heights, within (4) in order to obtain $k(z)$. In the above, $L$ is the Obukhov length, $\Delta u_{*} / u_{*}$ is the normalized stability-induced perturbation of friction velocity, and the subscripts 'off' and ' $r m s$ ' denote long-term mean ("offset" as labelled in the EWA ${ }^{4}$ ) and root-mean-square quantities. The height scale $z_{m}$ depends on roughness, and is typically 70-90 m (with larger values over rougher surfaces), and is discussed further below (also see Appendix); the coefficients $\left[\Delta u_{*} / u_{* 0}\right]$ are of order 0.1 , and the stability corrections follow classic Monin-Obukhov forms for stable $\left(\psi_{s}\right)$ and unstable $\left(\psi_{u}\right)$ conditions (Troen and Petersen 1989). We note that the above ratio is not a true wind profile, but is instead designed to give optimal $\left\langle U^{3}(z)\right\rangle$, by extrapolating measured $p(U)$ via Weibull- $A$ and $k$ together through $\langle U\rangle$ and $\sigma_{U}$.

\subsubsection{Models for Climatological $\sigma_{U}$}

Continuing to outline the EWA perturbation forms for later comparison with its $k(z)$ prediction, we note the EWA does not explicitly prescribe a profile $\sigma_{U}(z)$, but exploits Eq. 7 by calculating stability-induced perturbations

$$
\frac{\sigma_{U}(z)}{\sigma_{U 0}(z)}=1+\left[\frac{\Delta u_{*}}{u_{* 0}}\right]_{\mathrm{rms}}\left|1-\frac{z}{z_{m}} \frac{\ln \left(z_{m} / z_{0}\right)}{\ln \left(z / z_{0}\right)}\right|
$$

normalized relative to neutral conditions, where again the coefficient $\left[\Delta u_{*} / u_{* 0}\right]_{\mathrm{rms}}$ is significantly smaller than 1 (about 0.2 over land and $<0.1$ over sea; c.f. Troen and Petersen 1989). As evident in (10), $z_{m}$ was defined by the EWA to be the height of minimum stability-induced perturbations to the long-term wind variability.

2 Note Kelly and Gryning (2010) contains a typographical error in the definition of $s$, introduced after article proofing.

3 The inverse Obukhov length variability $\sigma_{ \pm}$is proportional to the ratio of long-term r.m.s. heat flux to $\left\langle u_{* 0}\right\rangle^{3}$, for either stable or unstable conditions; see Kelly and Gryning (2010).

${ }^{4} L_{\mathrm{off}}$ is typically positive in application, though in the case $L_{\mathrm{off}}<0$, the EWA takes $\psi_{S}=0$ in (9), and then $\psi_{u}$ becomes a function of $L_{\mathrm{off}}$ as well as $L_{\mathrm{rms}}$; see Troen and Petersen (1989) for more detail. 
'Tall profile' modelling of dimensionless wind-speed variance. We begin with a simple heuristic parametrization for the dimensionless long-term standard deviation of wind speed suggested by measurements,

$$
\frac{\sigma_{U}}{\sigma_{U_{0}}}=1+\frac{\left(n_{+} \sigma_{+} h_{\mathrm{eff}}\right)^{-1}}{\ln \left(z / z_{0 m}\right)}-\frac{1-z / h}{1+\left[\left(n_{+} \sigma_{+}^{-1} / \ell_{\mathrm{mid}}\right)^{2}+\left(n_{-} \sigma_{-}^{-1 / 3} / \ell_{\mathrm{mid}}^{1 / 3}\right)^{2}\right]^{1 / 2}},
$$

where $\sigma_{U_{0}}(z)$ is taken to be $\sigma_{u_{* 0}} \ln \left(z / z_{0}\right)$, implicitly following the EWA; here in Eq. 11 the notation echoes that used in the probabilistic wind-profile expression (8). We assume Weibull-distributed $u_{* 0}$ to obtain, for example, $\sigma_{u_{* 0}} \approx 0.5\left\langle u_{* 0}\right\rangle$ near $k=2$ via Eq. 2 .

A second model for the long-term wind variance arises through consideration of the square of the wind profile. Just as Kelly and Gryning (2010) found the mean wind profile (8) by integrating the wind profile (their Eq.10) over $L^{-1}$, here we start with

$$
\left\langle\left(\frac{\kappa U}{u_{* 0}}\right)^{2}\right\rangle(z)=\iiint\left[\frac{\kappa U}{u_{* 0}}\left(z, z_{0}, L^{-1}, h\right)\right]^{2} \mathbb{P}\left(L^{-1}, h, z_{0}\right) \mathrm{d} L^{-1} \mathrm{~d} h \mathrm{~d} z_{0} .
$$

As a first attempt we assume independence of $L^{-1}$ and $h$ and a relatively narrow long-term roughness distribution (i.e. $z_{0} \rightarrow z_{0 m}$ ) to reduce the joint probability distribution function $\mathbb{P}\left(L^{-1}, h, z_{0}\right)$ in $\left(12\right.$ a) to $P\left(L^{-1}\right) P(h)$, to obtain

$$
\left\langle\left(\frac{\kappa U}{u_{* 0}}\right)^{2}\right\rangle(z) \approx \iint\left[\ln \left(z / z_{0 m}\right)-\frac{z}{h} \psi_{e}\left(a z L^{-1}\right)\right]^{2} P\left(L^{-1}\right) \mathrm{d} L^{-1} P(h) d h .
$$

Here the effective stability function $\psi_{e}\left(z L^{-1}\right) \approx-4 z L^{-1}$ approximates the effective 'blending' of the total stability contributions from $\psi(z / L)$ at $z=z_{0 m}$ up to $\overline{\psi(z / L)}{ }^{z} \approx \psi(z / 2 L)$ at $z=h$. Adopting the generalized Gamma-distribution form

$$
P(h)=\left(\frac{h}{\sigma_{h}}\right)^{p-1} \frac{e^{-h / \sigma_{h}}}{\sigma_{h} \Gamma[p]}
$$

used by Liu and Liang (2010) to fit observation-derived ABL depths compiled over a number of extensive independent campaigns, with (3) we then find a profile of the long-term standard deviation of wind speed behaving approximately as

$$
\sigma_{U} \approx \sigma_{u_{* 0}} \sqrt{\left\langle\frac{\kappa U}{u_{* 0}}\right\rangle(z ; \ldots) \ln \left(\frac{z}{z_{0 m}}\right)-\left(\frac{c_{h} z}{h_{\mathrm{eff}}}\right)\left\langle\psi_{e}\right\rangle(z)+n_{+}\left(7.2 \sigma_{+} z\right)^{2}} .
$$

where $\left\langle\kappa U / u_{* 0}\right\rangle$ is as shown in (8) and $h_{\text {eff }}$ is the mean ABL depth following (13). Here $c_{h}$ is found to be approximately $3-5$ and in effect accounts for variability in $h,{ }^{5}$ and the constant of 7.2 arises from the integration using $P\left(L^{-1}\right)$ from Kelly and Gryning (2010).

The $\sigma_{U}$ profile implied by the EWA form (10) is shown in Fig. 2, along with the heuristic model (11) and reduced statistical model (14), for one of the three cases we consider here: one year of sonic anemometer data from the suburban Hamburg tall tower (detailed in Gryning et al. 2007), with wind directions limited to the relatively homogeneous 'residential' sector (as in Kelly and Gryning 2010). One can see that relatively good predictions for the long-term windspeed variability are afforded by the models developed here, compared to the EWA. Similar

5 The factor $c_{h}$ can be recovered by integrating with the generalized form (13) of $P(h)$ fit to aggregated measurements of $h$ by Liu and Liang (2010); this is e.g. roughly 3-5 over land, using their values of $p$ and $\sigma_{h}$, due to stable conditions dominating the statistics of $1 / h$. 


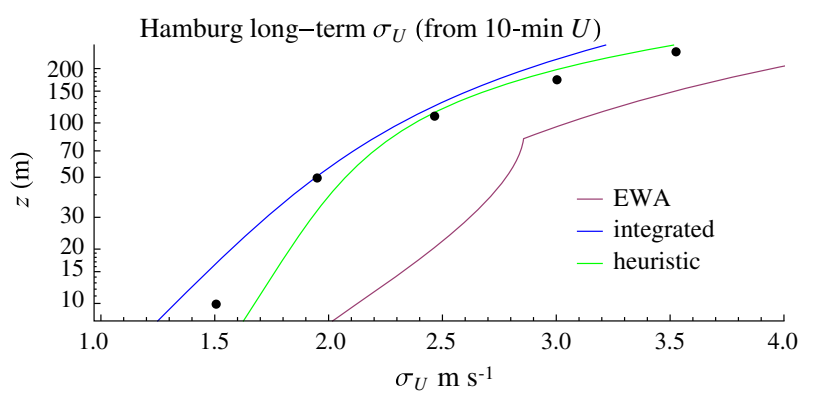

Fig. 2 Measured (dots) and modelled $\sigma_{U}$, Hamburg case. Magenta is EWA form (10), green is heuristic model (11), and blue is reduced probabilistic stability model (14)

results are obtained for the other two cases that we examine below (Høvsøre and Cabauw, c.f. Kelly and Gryning 2010), though the integrated approximation (14) gives poorer results for the Cabauw case, and systematically overpredicts $\mathrm{d} \sigma_{U} / \mathrm{d} z$ in the surface layer; the latter is evident in Fig. 2. Notable is that the form (14) based on the statistical profile, which contains the influence of variations in both stability and ABL depth, reproduces the change in $\mathrm{d} \sigma_{U} / \mathrm{d} z$ typically observed around $100-150 \mathrm{~m}$. Again we will not directly use dimensional $\sigma_{U}(z)$ to predict profiles of Weibull-statistics, but rather the dimensionless forms of (10), (11) and (14) to do so, via the ratio of $\sigma_{U}$ as seen in Eq. 7; thus the $\sigma_{u_{* 0}}$ from Eq. 14 will disappear in the calculation of $k(z)$, making the result insensitive to any assumption about the distribution of friction velocity or $\sigma_{u_{* 0}}$.

Also evident in Fig. 2 is a non-physical kink in the $\sigma_{U}$ profile implied by the EWA formulation (10), though we are reminded that it was defined as a normalized perturbation intended to extrapolate $k$ from one height to another, and is not an explicit profile. By design the kink at height $z_{m}$ will determine the maximum in $k(z)$, as shown below. Above $z_{m}$, the behaviour prescribed by the EWA form (10) might appear somewhat arbitrary, but it is again cast as a perturbation growing away from $z=z_{m}$, and the coefficient of the height-dependent part is damped in application (e.g. the WAsP software) by an exponentially decreasing form to avoid spuriously large values. ${ }^{6}$

\section{Weibull- $k$ Profile Models and Results}

\section{1 $k$-Profiles Resulting from Probabilistic Wind-Speed and Variance Profiles}

Figure 3 displays $k(z) / k\left(z_{\mathrm{obs}}=50 \mathrm{~m}\right)$ calculated via (7), using the tall wind profile (Eq. 8 ) in concert with both of the new long-term standard deviation wind-profile forms (11) and (14) for sites at Høvsøre, Hamburg, and Cabauw. The data from Høvsøre consisted of five years of wind profiles from the homogeneous land sectors, Hamburg has one year of residential sectors, and Cabauw has two years of the homogeneous south-west sector. The winds, fluxes and subsequent stability parameters used in our models were measured by sonic anemometers in the surface layer, with more details of the data found in Kelly and Gryning (2010) and Gryning et al. (2007). Figure 3 also shows the EWA result for these cases, based

6 The EWA implementation WAsP uses a factor $\exp \left(-z / z_{G}\right)$ to damp its linearized stability perturbations of wind statistics, where $z_{G}=\left(u_{* G} / f\right) \exp \left(-A_{0}\right) \approx u_{* G} /(6 f)$ and $A_{0}=1.8$ is the streamwise geostrophic drag-law constant. 

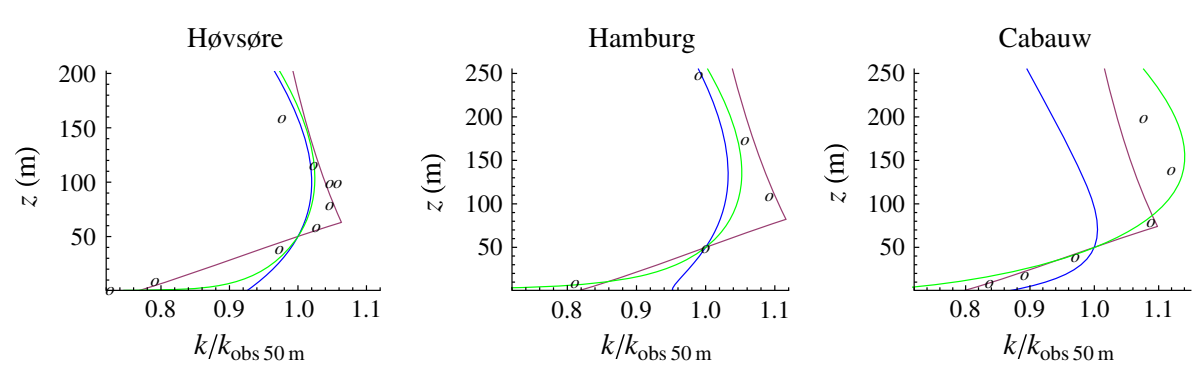

Fig. 3 Modelled normalized Weibull parameter profile $k(z) / k_{\mathrm{obs}}(z=50 \mathrm{~m})$ for homogeneous land sectors (c.f. Kelly and Gryning 2010), based on 40-60 m interpolated $k$ at Høvsøre, $k(50 \mathrm{~m})$ at Hamburg, and 40-80 m interpolated $k$ at Cabauw, respectively. Magenta using EWA forms (9-10); green via the $\sigma_{U}$ model (11); blue using (14) for $\sigma_{U}$. Calculations via both Eqs. 11 and 14 used measured surface momentum and heat fluxes from each site. Circles observations

on (9-10), as well as the observed normalized $k(z)$. As shown in the figure, the heuristic empirical formulation (11) for $\sigma_{U}$ leads to relatively good results, comparable to or better than the EWA in the 'tall' regime, i.e. for $z \gtrsim 100 \mathrm{~m}$ for the three sites considered. In the ASL, particularly below $z_{m}$ (less than $80 \mathrm{~m}$ over land), the EWA model gives a better performance. This is again consistent with the success (standard use) of the EWA method in wind energy in past decades, for turbines over flat land with hub heights in this height range.

The reduced probabilistic model (14) gives poorer $k(z)$ predictions than those using either the heuristic or EWA model. In the ASL, this is primarily due to over-prediction of $\mathrm{d} \sigma_{U} / \mathrm{d} z$ and subsequent under-prediction of $\sigma_{U}$ near the surface, which leads to under-prediction of $\mathrm{d} k / \mathrm{d} z$ in the ASL and over-prediction of $k$ near the surface when extrapolating away from observations at $50 \mathrm{~m}$. This is due in part to the simplifications used to obtain a closedform expression for $\sigma_{U}$ based on the stability distribution $P\left(L^{-1}\right)$; improvement of such is expected to lead to better $k(z)$. However, what is significant with the use of the new probabilistic derivation (14) is the qualitative, physical result that the mutual contributions of $\sigma_{h}$ and the surface influences $z_{0}$ and $\sigma_{1 / L}$ together determine the bowed shape of $k(z)$ : the top-down statistical influence of ABL (through $c_{h}$ from $P(h)$ ) and bottom-up contribution from surface-based variability (via $P\left(L^{-1}\right)$ here) help to physically explain the Weibull-k profile.

Despite the EWA model being based on the idealized perturbation-based expression (10), which gives rise to a non-physical kink, neither new model for $\sigma_{U}(z)$ leads to consistently better predictions of $k(z)$ at the sites considered, despite the improved predictions of $\sigma_{U}$ profiles as shown in Fig. 2; thus we consider an alternative method for observation-based prediction of $k(z)$ below.

\subsection{Alternative Weibull- $k$ Profile}

Due to the limited performance of the $\sigma_{U}$-based models introduced above, we turn to another method to predict $k(z)$ from observations at an arbitrary height, following from the empirical parametrization

$$
k(z)=k_{\mathrm{sfc}}+c_{k}\left(z-z_{\mathrm{sfc}}\right) e^{-\left(z-z_{\mathrm{scc}}\right) /\left(z_{r}-z_{\mathrm{scc}}\right)}
$$

of Wieringa (1989). Here the 'reversal height' $z_{r}$ is the height of maximum $k$ (corresponding to maximum $\left.\langle U\rangle / \sigma_{U}\right)$, with $k_{\mathrm{sfc}} \equiv k\left(z_{\mathrm{sfc}}\right)$ the near-surface value of $k, z_{\mathrm{sfc}} \approx 10 \mathrm{~m}$, low enough to be contained within the shallowest (nocturnal) boundary layer such that it 
is climatologically representative. Investigating the various data in Wieringa (1989) and a number of other sites including those considered here, we find that Wieringa's constant $c_{k}$ corresponds to $k_{\mathrm{sfc}} /\left(z_{r}-z_{\mathrm{sfc}}\right)$ and has dimensions of $\mathrm{m}^{-1}$. Thus the parametrization (15) can be updated to be more self-consistent, without the arbitrary constant $c_{k}$ and with the proper 'dimensions', using the form

$$
k(z)=k\left(z_{\mathrm{sfc}}\right)\left[1+\xi\left(z, z_{r}\right) e^{-\xi\left(z, z_{r}\right)}\right]
$$

where $\xi \equiv\left(z-z_{\mathrm{sfc}}\right) /\left(z_{r}-z_{\mathrm{sfc}}\right)$. Noting that $k(z)$ may be affected well above the ASL by 'top-down' processes not directly driven by the surface, Eq. 16 can be further generalized with a form such as

$$
k(z)=k_{\mathrm{sfc}}\left[1+\xi\left(z, z_{r}\right) e^{-\xi\left(z, z_{r}\right)}\right]+\left(k_{h}-k_{\mathrm{sfc}}\right) f_{k}\left(z, z_{r} \ldots\right) .
$$

Here $k_{h}$ is taken to be the asymptotic value of $k$ as $z$ approaches and exceeds the climatological ABL depth $h_{\text {eff }}$, and $f_{k}\left(z, h_{\text {eff }}, z_{r} \ldots\right)$ is a profile function that dictates the transition towards $k_{h}$ approaching the ABL top. Simple useful forms for $f_{k}$ may include e.g. $z / h_{\text {eff }}$ or $1-\mathrm{e}^{-z / z_{r}}$, where $f_{k}$ is zero approaching the ground and $f_{k} \rightarrow 1$ as $z \rightarrow h_{\text {eff }}$. Gryning et al. (2013) found limited success modelling $k(z)$ by including an upper ABL influence, for a site with lidar measurements extending above the ABL.

However, to ensure (16) or (17) is usable for arbitrary $z$ within the range typically measured in the ABL (e.g. in wind energy applications, $z \leq 100 \mathrm{~m}$ ), i.e. above the shallowest ASL, one must translate observed $k$ to $k_{\text {sfc }}$. Without information about $k_{h}$, using the simple modelling choice $k_{h}=k_{\mathrm{sfc}}$ and adaptation of (16) gives

$$
k(z)=k\left(z_{\mathrm{obs}}\right) \frac{1+\xi\left(z, z_{r}\right) e^{-\xi\left(z, z_{r}\right)}}{1+\xi\left(z_{\mathrm{obs}}, z_{r}\right) e^{-\xi\left(z_{\mathrm{obs}}, z_{r}\right)}}
$$

where $z_{\mathrm{obs}}$ is the height of observation, and $z_{\mathrm{sfc}}$ is a near-surface height (within the ASL), nominally taken to be 5-10 m following Wieringa (1989). However, we find that choosing $z_{\mathrm{sfc}}=0$ gave optimal results for all the sites we analyzed, which leads us to remove this potentially uncertain 'extra' parameter; thus the observation-based empirical relation reduces to

$$
k(z)=k\left(z_{\mathrm{obs}}\right) \frac{1+\left(z / z_{r}\right) e^{-\left(z / z_{r}\right)}}{1+\left(z_{\mathrm{obs}} / z_{r}\right) e^{-\left(z_{\mathrm{obs}} / z_{r}\right)}} .
$$

However, the so-called 'reversal height' $z_{r}$ still needs to be prescribed.

\subsubsection{Reversal Height and Height of Minimum Variability in Wind Speed}

Troen and Petersen (1989) defined a height of minimum stability-induced variations $z_{m}$ based on first-order perturbations to the geostrophic drag law, describing where the minimum in $\sigma_{U}(z)$ occurs, which is in effect equal to the height of maximum $k$ and thus equivalent to $z_{r}$. However, the constant of proportionality reported in Troen and Petersen (1989) for $z_{m}$ is inappropriately small for this purpose, inconsistent with the constants used earlier in their derivation and also implying reversal heights smaller than observed (though it is chosen to give optimal $k(z)$ based on the EWA form (10) for dimensionless long-term wind variance). Re-deriving $z_{m}$ (see Appendix) gives the same expression for $z_{m}$ as in the EWA, but with a corrected coefficient, which we adopt for the reversal height,

$$
z_{r}=z_{m}=\alpha_{r} z_{0}\left(R o_{0}\right)^{0.9}
$$



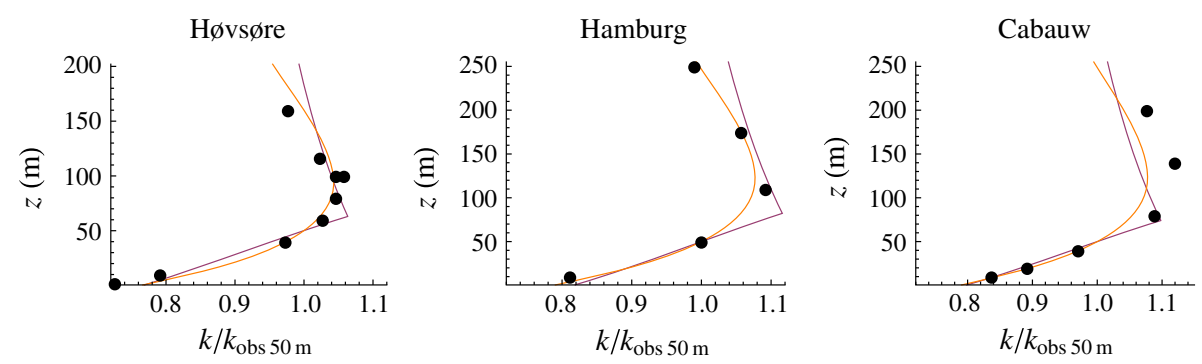

Fig. 4 Modelled Weibull parameter profile $k(z) / k\left(z_{\mathrm{obs}}=50 \mathrm{~m}\right)$ for Høvsøre, Hamburg, and Cabauw, wind sectors as in Gryning et al. (2007) and Kelly and Gryning (2010), using Eq. 19 with the corrected constant in (20). Maroon EWA result; orange (19); black dots data. Values of $k_{\mathrm{obs}} 50 \mathrm{~m}$ are 2.6, 2.3, and 2.55, respectively

with $\alpha_{r} \approx 0.003$, and where the 'surface' Rossby number is defined as $R o_{0}=G /\left(f z_{0}\right)$ and the corrected constant of 0.003 (formerly 0.002) is derived in the Appendix.

Figure 4 shows $k(z) / k(50 \mathrm{~m})$ versus $z_{\text {rec }}$ for the Høvsøre, Hamburg, and Cabauw cases, comparing the results of using the new formulation (19-20) with results from the EWA method. The figure shows that (19-20) give a robust performance at different sites, without the need for tuning. However, as seen above $z=200 \mathrm{~m}$ at Cabauw, the new adapted formulation for the Weibull- $k$ profile may be degraded by its lack of explicit parametrization for $k(z)$ at heights approaching the mean ABL depth, and might be improved by modifying the formulation to include $k_{h}$ as suggested in (17).

\subsection{Partitioning of Long-Term Wind Variance and Surface-Layer $k(z)$}

To gain insight into the profile of the Weibull- $k$ parameter, given its sensitivity to the profile of $\sigma_{U}$, we consider contributions to $\sigma_{U}(z)$. As with $U(z)$, one may attempt to partition the variance of wind speed into a component $\left(\sigma_{U}^{2}\right)_{\text {sfc }}$ due to surface heat fluxes (typically assumed to be a diurnal influence) and some 'baseline' variations $\sigma_{U}^{2} \equiv \sigma_{b}^{2}+\left(\sigma_{U}^{2}\right)_{\text {sfc }}$, which are presumed to be caused by mechanisms acting non-locally (over length scales $\gg z$ ) and with time scales $\gg 1$ day. This is in potential contrast to Wieringa (1989), who assumed $\sigma_{U}^{2} \approx\left(\sigma_{U}^{2}\right)_{\text {diurnal }}+\left(\sigma_{U}^{2}\right)_{\text {synoptic }}$ for heights below $80-100 \mathrm{~m}$ and described the behaviour of $k$ in terms of the diurnal component of $\sigma_{U}$.

It is expected that the ratio of diurnally-affected $\sigma_{U}(z)$ to the remainder of $\sigma_{U}$ may give an indication of the influence of surface-based stability effects. To this end the long-term spectra of wind speed were calculated, and band-pass filtered to both isolate the diurnal signal from the longer-term (e.g. synoptic and seasonal) signal as well as eliminate any dependence upon the length of time series. This was done at all observation heights for the Høvsøre, Hamburg, and Cabauw masts, using the overland sectors considered above (c.f. Gryning et al. 2007; Kelly and Gryning 2010). The spectral result is given in Fig. 5a for Høvsøre, which shows no clear "spectral gap" between diurnal and synoptic contributions, though one can see the hint of a gap for the near-surface $(10 \mathrm{~m})$ observations. We note this occurs despite the long-term near-surface heat-flux spectra (from the sonic anemometer at $10 \mathrm{~m}$ ) exhibiting a dominant diurnal peak (not shown) contributing roughly $90 \%$ of the heat flux. The upper cut-off frequency separating synoptic and diurnal components is approximately $0.8 \mathrm{day}^{-1}$, which also corresponds roughly to the minima seen in the spectra, and the lower cut-off corresponded to a time scale of roughly 40 days.

The second plot (Fig. 5b) displays the ratio of low-frequency $\left(\sigma_{\text {syn }}\right)$ to the diurnal component $\left(\sigma_{\text {diurnal }}\right)$ of the long-term standard deviation of wind speed for the three sites, and 

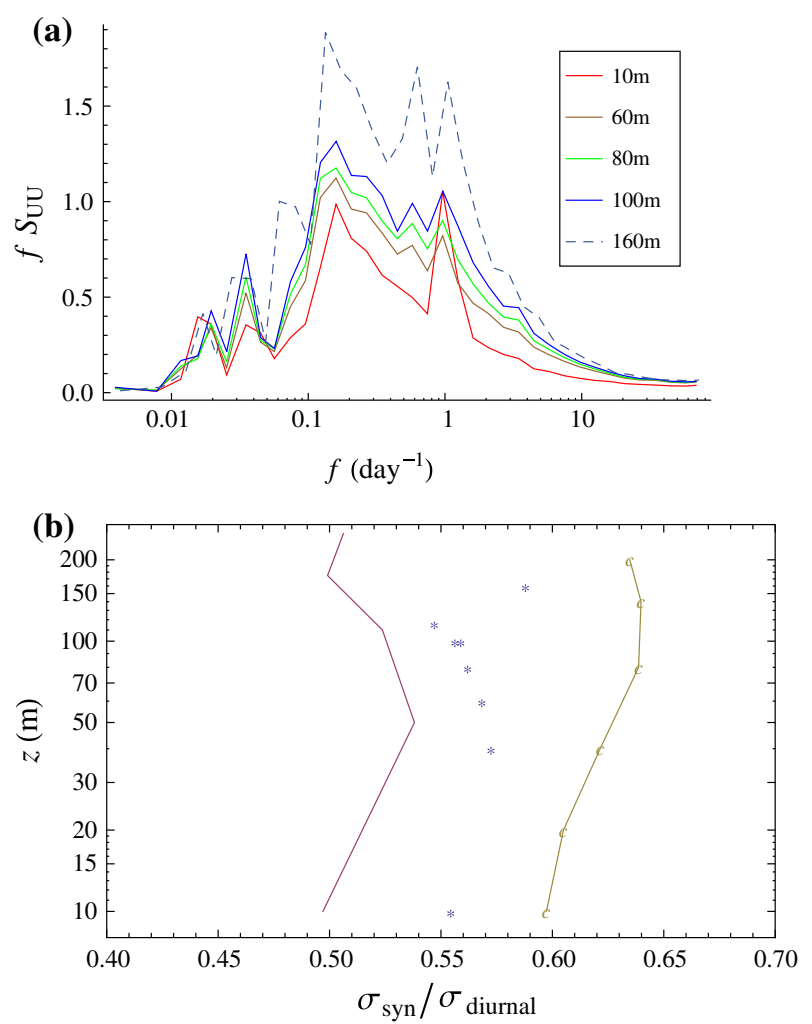

Fig. 5 a Long-term spectra of wind speed at various heights, Høvsøre homogeneous land sectors. b Ratio of synoptic to diurnal part of $\sigma_{U}$ for Høvsøre (stars), Hamburg (magenta), and Cabauw (beige), land sectors as in previous figures. $\sigma_{\text {syn }}$ is calculated between cut-off frequencies of 0.025 and 0.8 day $^{-1}$

shows no remarkable height dependence in the magnitude of diurnal wind-speed fluctuations relative to the longer time wind-speed fluctuations. The relative contribution of the diurnal component can be seen to decrease through the ASL and up to heights of 40-100m (depending on the site), with the ratio $\sigma_{\text {syn }} / \sigma_{\text {diurnal }}$ reaching a maximum, consistent with the decreasing influence of the surface. But an increasing short-term contribution is seen again at the highest level observations, suggesting a change in the behaviour of the wind distribution. One reason may be the increasing incidence of observations being taken near the capping inversion of the ABL; the ABL depth has a diurnal component due to the nighttime occurrence of reduced ABL depth, and the impact of inertial oscillations (and subsequent lower-level jets) with time scales $<1$ day are felt in the upper ABL (Baas et al. 2009). More data at higher levels are needed to investigate further, and importantly to determine if e.g. the synoptic component becomes dominant and predictable for heights falling in the upper portion of the distribution for $\mathrm{ABL}$ depth at a given site. At any rate, the variation of $\sigma_{\text {syn }} / \sigma_{\text {diurnal }}$ with height is relatively mild for the cases examined, with less than $10 \%$ variation over $2 \mathrm{~m}<z \lesssim 250 \mathrm{~m}$. This justifies to a great extent the attempt (Wieringa 1989) to use $\sigma_{U \text { diurnal }} /\langle U\rangle$ to describe $k(z)$.

\subsubsection{Surface-Layer Behaviour}

In the surface layer, for a surface heat-flux influence dominated by the diurnal contribution (especially true over land), the decrease in the diurnal component of long term wind-speed 
variance with height (as seen by the increase of $\sigma_{\text {syn }} / \sigma_{\text {diurnal }}$ in Fig. $5 b$ ) is consistent with the positive $d k / d z$ observed. For a given observation height in the surface layer, the EWA (Troen and Petersen 1989) ratios of normalized variance and mean wind speed can be reduced to give

$$
\begin{aligned}
\left.\frac{k(z)}{k_{\mathrm{obs}}}\right|_{\mathrm{ASL}} & \approx \frac{f_{k}\left(z, z_{0}\right)}{f_{k}\left(z_{\mathrm{obs}}, z_{0}\right)}, \\
f_{k}\left(z, z_{0}\right) & =\frac{1+\left[\Delta u_{*} / u_{* 0}\right]_{\mathrm{off}}}{1+\left[\Delta u_{*} / u_{* 0}\right]_{\mathrm{rms}}}+\frac{z}{\lambda\left(z_{0}\right)},
\end{aligned}
$$

where $\lambda\left(z_{0}\right)$ is a nearly logarithmic function of roughness length, varying from about $150 \mathrm{~m}$ over water to $350 \mathrm{~m}$ or more for $z_{0}>1.5 \mathrm{~m}$. With virtually no height-dependence in $\lambda$, the EWA predicts approximately constant $d k / d z$ throughout the ASL. Over land, using the nominal EWA mean and r.m.s. values of 0.07 and 0.18 for $\left[\Delta u_{*} / u_{* 0}\right]$ in (21), the ASL $k$-profile becomes approximately

$$
\left.k(z)\right|_{\mathrm{ASL}, \text { land }} \approx k_{\mathrm{sfc}}\left[1+\frac{z}{0.79 \lambda\left(z_{0}\right)}\right]
$$

where $k_{\mathrm{sfc}}$ is the near-surface value of $k$. This is in part responsible for the historically good performance of the EWA (via the commonly-used wind-industry software WAsP, Mortensen et al. 2001) for heights $z \lesssim 50 \mathrm{~m}$ over simple terrain; i.e. Eq. 21 replicates the roughnessdependent, nearly constant $d k / d z$ typically seen throughout the surface layer, also evident at the three sites shown in Fig. 4.

For comparison, when $k_{\mathrm{sfc}} \approx 2$, the $d k / d z$ values given by Wieringa (1989), who did not include roughness as an influence, correspond to EWA overland ASL values (cf. Eq. 22 of $k_{\mathrm{sfc}} /\left(0.79 \lambda\left(z_{0}\right)\right)$ with $z_{0} \approx 0.05 \mathrm{~m}$; the Wieringa (1989) model tends to give $d k / d z$ in the ASL that is representative of moderately rough land surfaces. This becomes a significant problem over the sea, where the lack of treatment of surface influence can result in an over-prediction of $d k / d z$ and subsequently poor extrapolations of $k$ from near-surface observations.

\section{Summary and Conclusion}

\subsection{Long-Term Variance and Subsequent Weibull Parameters}

The heuristic and simplified probabilistic models developed here for long-term wind-speed variance tend to give better agreement with observations well above the surface layer, compared to their EWA counterpart. Despite this, the new models for $\sigma_{U}(z)$ did not lead to improved predictions of $k(z)$ at the three sites considered: improved 'tall' profiles of windspeed variance (with slightly better mean wind-speed profiles), which use measured statistics of near-surface heat and momentum fluxes, do not necessarily lead to better profiles of the Weibull- $k$ parameter. The EWA perturbation expression for $\sigma_{U}$, together with its perturbation expression $\Delta U$, produce more consistent predictions of $k(z)$. Though the EWA expressions for scaling the mean wind speed and variance appear to imply unrealistic profiles, each of which do not match measurements very closely, these are coupled perturbation forms that together lead to good extrapolation of the Weibull- $k$ parameter, particularly in the surface layer. This is perhaps not surprising, since the EWA method was designed for the prediction of wind power, i.e. $\left\langle U^{3}\right\rangle$, using ASL theory and measurements. The relatively poorer $k(z)$ predictions given by the new (and better-performing) probabilistic $\sigma_{U}(z)$ formulation may 
also be due in part to the limited ability of $\left\langle U^{2}\right\rangle$ to accurately represent the Weibull- $k$ parameter; typically $k$ is estimated from a Weibull fit to an observed wind distribution via the third moment of wind speed, as the tails are not as well represented by the second moment as they are by $\left\langle U^{3}\right\rangle$. However, this is a compromise justified by $\left\langle U^{2}\right\rangle$ being more easily connected to the flow dynamics, as well as to its reduced demands on data and consequent uncertainty.

However, despite the limitations of the probabilistic formulation for $\sigma_{U}(z)$ presented here, an important conceptual finding follows from the $k(z)$ result: when using the probabilistic $\langle U\rangle / \sigma_{U}$ (which was derived consistent with Monin-Obukhov theory), one sees that the reversal height $z_{r}$, again where $k$ has its maximum, is determined by the distribution of ABL depth $P(h)$ together with the surface roughness and stability distribution. As far as the simplified probabilistic description (14) for $\sigma_{U}(z)$ can be trusted, the variability in ABL depth and in stability together lead to a maximum in $k(z)$ of approximately one-fifth to one-third of the effective (long-term) ABL depth. In other words the reversal height $z_{r}$, and thus the heightdependent variation of the shape of the wind distribution, is determined to some extent by the widths of the distributions of ABL depth $P(h)$ and stability $P\left(L^{-1}\right)$, particularly during stable conditions. The shallower ABL occurring during stable conditions leads to effectively larger values of $z / h_{\text {eff }}$ in the profile expressions, and the stable-stratification variability $\left(\sigma_{+}\right)$ has more influence on the profiles than does the unstable-condition variability $\left(\sigma_{-}\right)$; the latter follows from the stronger influence (height dependence) of stable stratification on wind speed (as evidenced by Monin-Obukhov theory). Some assumptions have been made in deriving $\sigma_{U}(z)$, which are of limited validity; current work includes the derivation of more applicable analytic representation of $\sigma_{U}$ via the distributions $P\left(L^{-1}\right)$ and $P(h)$, and refinement of the statistical theory describing the reversal height. Inclusion of the joint behaviour (correlation) of $L^{-1}$ and $h$ is currently lacking in our model description, and is a topic of continuing study as well.

\subsection{Different Profile Forms of the Weibull Shape Parameter}

We have adapted the EWA (Troen and Petersen 1989) concept of the height $z_{m}$ of minimum stability-induced deviations of the wind profile via a perturbed-geostrophic drag lawcorrecting a constant in its formulation-to apply $z_{m}$ as the reversal height $z_{r}$ where the profile of the Weibull- $k$ parameter reaches a maximum. We use the corrected $z_{m}=z_{r}$ as one improvement in a semi-empirical model for $k(z)$ building upon that of Wieringa (1989). Our model is by design extended to be amenable to measurement-based extrapolation, and is universal in the sense that it eliminates the effectively dimensional Wieringa (1989) 'constant,' which varies in an undetermined way from site to site.

While the probabilistic modelling for $U(z)$ and $\sigma_{U}(z)$ implies that $z_{r}$ depends on roughness and an effective boundary-layer depth, the geostrophic perturbation form for $z_{m}$ depends on roughness, geostrophic wind speed $G$, and latitude via $f$. These can be rectified by considering that the probabilistic formulations also depend on $G$ and $f$ via $\langle U\rangle$, and because the long-term characteristic ABL depth from the probabilistic model, affected by neutral and stable conditions, tends to be (to first order) proportional to the geostrophic scale surface friction velocity $u_{*}$ (Zilitinkevich et al. 2012) and thus to the geostrophic wind speed, due to the general existence of the drag law relating $G$ and $u_{*}$.

The assumption of Wieringa (1989) relating diurnal variance to $k$ is somewhat justified by our spectral analyses of the sites considered, since we find that the partitioning between synoptic and diurnal variability does not change significantly with height. This assumption, however, is not expected to hold in regions affected by e.g. sea breezes or tropical regimes with stronger diurnally-related forcing. The data considered here were taken over relatively 
simple terrain in areas lacking such mechanisms. While the adapted semi-empirical model (19) for the Weibull- $k$ profile has produced better observation-based extrapolations than the EWA method or the simple Wieringa (1989) form, it and the Wieringa (1989) model lack surface roughness in their parametrization. In particular such models give spuriously large $d k / d z$ in the surface layer over water, leading to poor extrapolations of $k$ when heights $<z \approx 70 \mathrm{~m}$ are involved, in contrast to the EWA (Troen and Petersen 1989) method.

Continuing work includes correcting the semi-empirical $k(z)$ model for surface roughness, and exploration of stability effects upon it. Given the failure of the probabilistic model and its assumption of independent probability distributions $P(L)$ and $P(h)$, this aspect is also under investigation to improve the $\sigma_{U}$ model and resultant $k(z)$. Further concurrent work includes better incorporation of expected ABL depth distributions $P(h)$ in the theory, particularly for improvement of Weibull- $k$ profiles obtained via profiles of long-term second moment of the wind speed along with the mean wind speed itself.

Acknowledgments We thank Burghard Brümmer at University of Hamburg for the Hamburg data, and KNMI and Dr. Fred Bosveld for the use of Cabauw mast data. We further acknowledge the numerous helpful comments and suggestions made by the reviewers, which improved the quality of the manuscript.

Open Access This article is distributed under the terms of the Creative Commons Attribution License which permits any use, distribution, and reproduction in any medium, provided the original author(s) and the source are credited.

\section{Appendix}

\section{Perturbations Around Neutral Geostrophic Drag Law}

Here we include an updated derivation of the height of minimum deviations from the basic geostrophic drag law and logarithmic wind profile, which is congruent with the 'reversal height' $z_{r}$ of the Weibull- $k$ profile and the change in slope observed in long-term $\sigma_{U}$ profiles. The theory includes derivation of the perturbation of the geostrophic drag law, which leads to the stability theory used in the European Wind Atlas (Troen and Petersen 1989) and justification of the constant $\alpha$ chosen in our modelling.

The EWA (Troen and Petersen 1989) estimates the geostrophic-scale perturbation $\mathrm{d} u_{*}$ due to small perturbations in the barotropic geostrophic resistance-law constants $A_{0}$ and $B_{0}$, which are in turn caused by first-order perturbations $\mathrm{d} H$ in geostrophic-scale heat flux (from neutral, i.e. around $H=0)$. This is done via the stability parameter $\mu \equiv \kappa\left(u_{*} / f\right) / L$, taking $\mathrm{d} A=\left(\mathrm{d} A_{0} / \mathrm{d} \mu\right) \mathrm{d} \mu$ and $\mathrm{d} B=\left(\mathrm{d} B_{0} / \mathrm{d} \mu\right) \mathrm{d} \mu$. Since $u_{*} / L=\left(\kappa g / T_{0}\right) H / u_{*}^{2}$, then

$$
\frac{d \mu}{\mu}=\frac{d H}{H}-2 \frac{d u_{*}}{u_{*}} .
$$

For a given $G$, and ignoring $\mathrm{d} z_{0}$, then the differential of the geostrophic drag law

$$
G=\frac{u_{* 0}}{\kappa} \sqrt{\left[\ln \left(\frac{u_{*} / f}{z_{0}}\right)-A_{0}^{2}\right]+B_{0}^{2}},
$$


can be written as

$$
\begin{aligned}
0 & =d G \\
& =G \frac{d u_{*}}{u_{*}}+\frac{u_{*} / \kappa}{\sqrt{\left[\ln \left(u_{*} / f z_{0}\right)-A_{0}\right]^{2}+B_{0}^{2}}}\left\{\left(\frac{d u_{*}}{u_{*}}-d A_{0}\right)\left[\ln \left(\frac{u_{*} / f}{z_{0}}\right)-A_{0}\right]+B_{0} d B\right\},
\end{aligned}
$$

or more succinctly as,

$$
\begin{aligned}
0 & =\mathrm{d} \ln G \\
& =\mathrm{d} \ln u_{*}+\left(\frac{u_{*}}{\kappa G}\right)^{2}\left\{\left(\mathrm{~d} \ln u_{*}+0.2 \mathrm{~d} \mu\right)\left[\ln \left(\frac{u_{*} / f}{z_{0}}\right)-A_{0}\right]+0.2 B_{0} \mathrm{~d} \mu\right\}
\end{aligned}
$$

if one takes $d B_{0} / d \mu=-d A_{0} / d \mu=0.2$ as in Troen and Petersen (1989). By rearranging (26) and exploiting (23), we obtain a relation between first-order perturbations in geostrophic friction velocity and heat flux,

$$
\mathrm{d} \ln u_{*}=\frac{0.2 \mu\left(\frac{u_{*}}{\kappa G}\right)^{2}\left\{B_{0}-\left[\ln \left(\frac{u_{*} / f}{z_{0}}\right)-A_{0}\right]\right\}}{1+\left(\frac{u_{*}}{\kappa G}\right)^{2}\left\{\left[\ln \left(\frac{u_{*} / f}{z_{0}}\right)-A_{0}\right](1-0.4 \mu)+0.4 \mu B_{0}\right\}} \mathrm{d} \ln H .
$$

Using the classic relation $\tan \varphi_{G}=B_{0} /\left[\ln \left(u_{*} / f z_{0}\right)-A_{0}\right]$ for the barotropic ABL (Zilitinkevich et al. 1967; Hess 2004), this becomes

$$
\mathrm{d} \ln u_{*} \approx 0.2 B_{0} \mu\left(\frac{u_{*}}{\kappa G}\right)^{2}\left\{1-\cot \varphi_{G}\right\} \mathrm{d} \ln H
$$

where $\varphi_{G}$ is the geostrophic turning angle. The EWA gives a form equal to $\mathrm{d} u_{*}=$ $c_{G}\left(g / \rho c_{p} T_{0} f G^{2}\right) \mathrm{d} H$, which is equivalent to (28) but with $0.2 B_{0}\left(1-\cot \varphi_{G}\right)$ replaced by a constant $c_{G}$ equal to 2.5 . This value is a little higher than that expected using the geostrophic drag law (as implemented in the EWA) in (27), which gives a constant between 0.44 and 2.0 over the range of roughnesses encountered in practice (water to urban/forest). However, given that the $\tan \left(\varphi_{G}\right)$ dependence is not appropriate for latitudes approaching the equator (Arya and Wyngaard 1974), and the uncertainty in the behaviour in $A(\mu)$ and $B(\mu)$ (e.g. Hess and Garratt 2002; Hess 2004), the lack of $\varphi_{G}$ dependence in the EWA form connecting $\Delta u_{*}$ and $\Delta H$ may be interpreted as a reasonable modelling choice.

\section{Height of Minimum Deviations from a Neutral Profile}

The EWA defines the height $z_{m}$ to be where first-order effects of surface heat-flux variations vanish. Taking the differential of the Monin-Obukhov wind profile to be zero there, $\left.\mathrm{d}\left\{u_{*}\left[\ln \left(z / z_{0}\right)-\psi(z / L)\right]\right\}\right|_{z=z_{m}}=0$, we have

$$
0=\mathrm{d} u_{*}\left[\ln \left(z_{m} / z_{0}\right)-\psi\left(z_{m} L^{-1}\right)\right]-u_{*}\left[\mathrm{~d} \ln H-3 \mathrm{~d} \ln u_{*}\right] L^{-1} \frac{\mathrm{d} \psi}{\mathrm{d} L^{-1}} .
$$

Using $\mathrm{d} \psi / \mathrm{d} L^{-1} \rightarrow-b z$ in the neutral limit $\mathrm{d} L^{-1} \rightarrow 0$, exploiting (28) and the definition of Obukhov length $L$ and using the stable side form for $\psi$, one then obtains

$$
\frac{z_{m}}{\ln \left(z_{m} / z_{0}\right)}=\frac{c_{G}}{b} \frac{u_{*}^{3}}{\kappa f G^{2}} .
$$

The EWA uses $c_{G}=2.5$, while here we have from (27) approximately $c_{G} \approx 0.2\left[B_{0}+A_{0}-\right.$ $\left.\ln \left(u_{*} / f / z_{0}\right)\right]$ or from $(28) c_{G} \approx 0.2 B_{0}\left(1-\cot \varphi_{G}\right)$. The EWA continues by using a reduced 
geostrophic drag law $\left(u_{*} / G\right) \approx 0.5 /\left[\ln \left(u_{*} / f / z_{0}\right)-A_{0}\right]$ (Jensen et al. 1984) in (30), arriving at an approximation for $(30)$ of $0.1(G / f) /\left[\ln \left(u_{*} / f / z_{0}\right)-A_{0}\right]^{3}$ and ultimately approximating this expression for $z_{m} / \ln \left(z_{m} / z_{0}\right)$ with a power law to obtain

$$
z_{m}=\alpha_{m} z_{0}\left(\frac{G}{f z_{0}}\right)^{0.9}
$$

where $\left.\alpha_{m}\right|_{\text {EWA }}=0.002$. However, such a choice for $\alpha_{m}$ implies a value of $b=8$, because the constant of 0.1 in the EWA approximation for (30) derives from $0.5^{3} c_{G} / b \kappa$, which is equal to 0.16 for $b=4.7$ and $c_{G}=2.5$. This implies one should have $\alpha_{m}=0.003$ for this value of $c_{G}$. The latter correct value of $\alpha_{m}$ indeed corresponds to the observed reversal heights, whereas using the EWA value of 0.002 gives $z_{m}$ between $65-80 \mathrm{~m}$. Such a choice for $\alpha_{m}$ might be noted to compensate for the erroneously large $c_{G}$ used in the EWA; however if $c_{G}$ is corrected then one would need to adjust the recommended ('default') values of $H_{\text {off }}$ and $H_{\mathrm{rms}}$ used in the EWA stability treatment.

Further, in application (e.g. in the commonly-used wind prediction software WAsP) the EWA takes $G$ to be at least $16 \mathrm{~m} \mathrm{~s}^{-1}$; for most observations the geostrophic drag law implies $G<16 \mathrm{~m} \mathrm{~s}^{-1}$, so (31) in effect reduces to $z_{m} \propto z_{0}^{0.1} f^{-0.9}$, and the $k$-profile has a maximum at a height dictated by roughness and latitude. While the $z_{m}$ values predicted by the EWA have been systematically low (due to $\alpha$ above), the EWA has achieved substantial success in application over several decades; however, this is likely due to the relatively small number of validated predictions above $80-100 \mathrm{~m}$. Thus the validity of the simplified $\left(z_{0}, f\right)$ dependence of $z_{m}$ has not (yet) been verified.

\section{References}

Akdağ SA, Dinler A (2009) A new method to estimate Weibull parameters for wind energy applications. Energy Convers Manag 50:1761-1766

Arya SPS, Wyngaard JC (1974) Effect of baroclinicity on wind profiles and the geostrophic drag law for the convective boundary layer. J Atmos Sci 32:767-778

Baas P, Bosveld FC, Baltink HK, Holtslag A (2009) A climatology of nocturnal low-level jets at Cabauw. J Appl Meteorol Climatol 48(8):1627-1642

Gryning SE, Batchvarova E, Brümmer B, Jørgensen H, Larsen S (2007) On the extension of the wind profile over homogeneous terrain beyond the surface boundary layer. Boundary-Layer Meteorol 124(2):371-379

Gryning SE, Batchvarova E, Floors R, Peña A, Brümmer B, Hahmann AN, Mikkelsen T (2013) Long-term profiles and Weibull distribution parameters up to 600 meters in a rural coastal and an inland suburban area. Boundary-Layer Meteorol 150(2):167-184

Hess GD (2004) The neutral, barotropic planetary boundary layer, capped by a low-level inversion. BoundaryLayer Meteorol 110:319-355

Hess GD, Garratt JR (2002) Evaluating models of the neutral, barotropic planetary boundary layer using integral measures. Part I: overview. Boundary-Layer Meteorol 104:359-369

Jensen NO, Petersen EL, Troen I (1984) Extrapolation of mean wind statistics with special regard to wind energy applications. World climate programme report, WMO, Geneva, report \# WCP-86

Justus C, Mikhail A (1976) Height variation of speed and wind distribution statistics. Geophys Res Lett 3(5):261-264

Justus C, Hargraves W, Mikhail A, Graber D (1978) Methods for estimating wind speed frequency distributions. J Appl Meteorol 17:350-353

Kelly M, Gryning SE (2010) Long-term mean wind profiles based on similarity theory. Boundary-Layer Meteorol 136(3):377-390

Lenschow DH, Mann J, Kristensen L (1994) How long is long enough when measuring fluxes and other turbulence statistics? J Atmos Ocean Technol 11:661-673

Liu S, Liang XZ (2010) Observed diurnal cycle climatology of planetary boundary layer height. J Clim 23:5790-5809

Mikhail A (1985) Height extrapolation of wind data. Solar Energy Eng 107:10-14 
Monin AS, Obukhov AM (1954) Basic laws of turbulent mixing in the surface layer of the atmosphere. $\mathrm{Tr}$ Akad Nauk SSSR Geofiz Inst 24:163-187

Mortensen NG, Landberg L, Rathmann O, Frank HP, Troen I, Petersen EL (2001) Wind atlas analysis and application program (WAsP). Report, Ris $\varnothing$ National Laboratory, Roskilde, Denmark

Spera DS, Richards TR (1979) Modified power law equations for vertical wind profiles. In: Proceedings of the 1979 Conference and Workshop on Wind Characteristics and Wind Energy Siting, Am Meteorol Soc, Boston, MA

Troen I, Petersen EL (1989) European Wind Atlas. Risø National Laboratory, Roskilde

Wieringa J (1989) Shapes of annual frequency distributions of wind speed observed on high meteorological masts. Boundary-Layer Meteorol 47:85-110

Zilitinkevich SS, Laikhtman DL, Monin AS (1967) Dynamics of the atmospheric boundary layer. Izv Atmos Ocean Phys 3:170-191

Zilitinkevich SS, Tyuryakov SA, Troitskaya YI, Mareev EA (2012) Theoretical models of the height of the atmospheric boundary layer and turbulent entrainment at its upper boundary. Izv Atmos Ocean Phys 48(1):133-142 